\title{
La necesidad de reinvertarse ante el reto de la cronicidad
}

\section{The need to redirect oneself facing the challenge of chronicity}

\section{J.J. Mira Solves}

Área de Psicología social

Universidad Miguel Hernández
Vivimos más y vivimos mejor durante más tiempo. Nuestro sistema sanitario ha sido capaz de proporcionarnos mayor esperanza de vida y, también, que podamos disfrutar de una mejor calidad de vida durante muchos más años.

Ahora bien, las proyecciones demográficas anuncian un sobre-envejecimiento de la población anciana, un incremento del número de hogares unipersonales entre los mayores y un notable crecimiento en el volumen de personas que sufren al mismo tiempo varias condiciones crónicas ${ }^{1}$.

Por esta razón, los sistemas sanitarios de los países desarrollados buscan cómo reinventarse ${ }^{2}$. Las soluciones que hasta ahora se han demostrado útiles, plantean más interrogantes que respuestas, y necesitamos alternativas a un modelo que en su origen fue diseñado para afrontar problemas de salud bien distintos a los actuales y en un contexto social, tecnológico y de relación entre pacientes, profesionales y sistema sanitario, muy diferente al que vivimos hoy día.

Convivimos en España más de 20 millones de enfermos crónicos. Un 76\% de las personas mayores de 65 años sufren, al menos, una enfermedad crónica. Y si avanzamos en edad, comprobamos que entre las personas mayores de 75 años es muy frecuente la multimorbilidad, sufriendo este segmento de población una media de 3,2 enfermedades crónicas. Estas cifran justifican que estemos destinando tres cuartas partes de los recursos sanitarios a paliar los efectos de la cronicidad. Y la previsión es que este porcentaje puede seguir incrementándose.

La insatisfacción con los resultados en términos asistenciales, con una atención de "puertas giratorias", en la que pacientes y profesionales se ven atrapados sin que podamos ofrecer una solución acorde a las necesidades reales; la sensación de que no podemos seguir prestando atención a las patologías crónicas de forma aislada, y sólo desde el sistema sanitario; junto a la impresión de que existen alternativas para reducir los efectos de la carga de la cronicidad; han propiciado en los 
últimos tres años una sucesiva presentación de planes y de propuestas de nuevos modelos de abordaje de la cronicidad en las comunidades autónomas y por parte del propio Ministerio de Sanidad. Se busca dar una respuesta, que sin ser radicalmente nueva, afronte a uno de los principales retos a los que debe hacer frente nuestro sistema sanitario.

La carga de la cronicidad, y de la multimorbilidad que un número creciente de personas afronta día a día ${ }^{3}$, obliga, al menos, a revisar nuestra definición de salud, el reparto de roles entre profesionales y pacientes y el significado de la atención integral. Muchos de estos enfermos gozan de una vida independiente y se afanan diariamente para llevar a cabo sus proyectos. Tenemos ante nosotros una realidad que nos pide reinventar nuestro modelo para prestar atención a los enfermos crónicos, extendiéndolo más allá de lo estrictamente sanitario.

La coordinación de los distintos niveles asistenciales para lograr que la persona se halle en su entorno natural el mayor número posible de años, junto a un cambio a la hora de entender el papel del paciente, han propiciado modelos que buscan una atención en la que los recursos sanitarios y comunitarios comparten objetivos, responsabilidades y la búsqueda de una forma común con la que medir los resultados en salud. Nuestro escenario debe cambiar para en vez de trabajar con enfermedades, trabajar con enfermos que tienen distintas necesidades en diferentes momentos de su ciclo vital.

Uno de los más claros exponentes de este enfoque es el Chronic Care $\mathrm{Model}^{4}$ (CCM) que ha logrado reunir a su alrededor un importante volumen de estudios, experiencias y evidencias de su utilidad como palanca desde la que hacer evolucionar el modelo de atención.

El CCM apuesta por una atención más proactiva, continuada, integrada y centrada en el paciente. Propone un modelo de atención en el que la salud es entendida como la capacidad que posee la persona para adaptarse y desenvolverse ante los retos físicos, mentales y sociales que el devenir de la vida conlleva. En definitiva, plantea el reto a los profesionales de ser capaces de promover competencias en el paciente que le permitan gobernar su vida con mayor autonomía durante el mayor tiempo posible.

El CCM nos propone algunas de las estrategias que ahora forman parte de las líneas de trabajo de los servicios de salud en nuestro país, como por ejemplo: que la multimorbilidad requiere una forma diferente de actuar; que el paciente debe participar de forma activa en su autocuidado; estratificar la población según su nivel de riesgo y sus necesidades de atención sanitaria y social para reorganizar el modo en que prestamos la atención; fomentar equipos multidisciplinares que trabajen de forma proactiva y continuada, integrando a todos los niveles y sistemas (no solo el sanitario) que se citan en un mismo territorio; prestar más cuidados en la comunidad y a domicilio y menos en los hospitales; o aprovechar mejor las tecnologías de la información y la comunicación para incrementar la capacidad del clínico, ayudándole a controlar síntomas y parámetros biológicos.

En España, la herramienta de evaluación de la atención a la cronicidad IEMAC $1.0^{5}$ se ha basado en este modelo y propone, para asegurar que los resultados sean sostenibles en el tiempo, abordar la enfermedad crónica de modo sistémico, combinando distintas intervenciones ligadas a diferentes dimensiones del CCM. 
El artículo de Orueta y $\operatorname{col}^{6}$, que se publica en este número de Anales del Sistema Sanitario de Navarra, se enmarca en este cambio de enfoque en el que se apuesta por el desarrollo de modelos de provisión integrada social y sanitaria y donde, en palabras de sus autores, la coordinación de servicios sociales y sanitarios a través de la conexión de sistemas de información y la teleasistencia, como plataforma de servicios, ofrecen oportunidades relevantes de innovación en la intervención sobre esta población.

Aunque no ha sido el único, el Departamento de Sanidad y Consumo del Gobierno Vasco, ha apostado, con su "Estrategia para afrontar el reto de la cronicidad en Euskadi”, por esta reinvención del modelo asistencial en Osakidetza ${ }^{7}$, buscando alternativas a la forma tradicional en la que se atendía a la cronicidad desde el sistema sanitario público.

En este caso, se presenta un estudio epidemiológico relizado sobre los ususarios del servicio Público de Teleasistencia de Euskadi, betiON, cuya titularidad corresponde al Departamento de Empleo y Políticas Sociales y es, por tanto, un ejemplo de integración sociosanitaria que se beneficia de la interconexión de los ámbitos social y sanitario, intercambiando información y proponiendo objetivos comunes, lo que potencia su capacidad más allá de lo que cada uno por su cuenta pudiera ser capaz de lograr.

Orueta y col presentan resultados que permiten conocer el perfil de los pacientes en teleasistencia, pero que ponen de manifiesto una realidad que requiere de respuestas distintas a las tradicionales. Este trabajo nos ofrece un ejemplo con su programa de teleasistencia y algunas pistas con las que diseñar intervenciones diferenciadas según las necesidades de distintos segmentos de la población y es aquí, probablemente, donde radica su mayor potencial.

La cronicidad, y la multimorbilidad que la acompaña, están con nosotros desde hace tiempo, pero ahora ocupan un lugar más destacado en la atención de políticos, gestores y profesionales. Esperemos que no sea solo una moda y que seamos, entre todos, capaces de reinventarnos.

\section{BIBLIOGRAFÍA}

1. Mathers CD, Loncar D. Projections of global mortality and burden of disease from 2002 to 2030. PLoS Med 2006; 3: e442.

2. Allotey P, Reidpath DD, Yasin S, et al. Rethinking health-care systems: a focus on chronicity. Lancet 2011; 377: 450- 451.

3. Tinetti ME, Fried TR, Boyd CM. Designing health care for the most common chronic condition-multimorbidity. JAMA 2012; 307: 2493-2494.

4. Wagner EH, Austin BT, Von Korff M. Organizing care for patients with chronic illness. Milbank Q. 1996; 74: 511-544.

5. Nuño-Solinís R, Fernández-Cano P, Mira JJ, Toro-Polanco N, Contel JC, Guilabert Mora M, Solas O. Desarrollo de IEMAC, un instrumento para la Evaluación de Modelos de Atención ante la Cronicidad. Gac Sanit 2013; 27: 128-134.

6. Orueta JF, Alonso-Morán E, Nuño-Solinis R, Alday-Jurado A, Gutiérrez-Fraile E, García-Álvarez A. Prevalencia de cronicidad y multimorbilidad en la población cub9erta por el servicio público de teleasistencia de Euskadi: impacto en costes y calidad de la atención. An Sist Sanit Navar 2013; 36: 429-440.

7. Nuño-Solinís R, Orueta JF, Mateos M. An answer to chronicity in the Basque Country: primary care-based population health management. J Ambul Care Manage 2012;35: 167-177. 
